\title{
Paroxysmal choreoathetosis as a presenting symptom in idiopathic hypoparathyroidism
}

\author{
D. S OF F E R, A. L I C H T, I Y A A R, A N D O. A B R A M S K Y \\ From the Department of Neurology and Nephrological Service, Hadassah University Hospital, \\ Jerusalem, Israel
}

SUMMARY A patient with idiopathic hypoparathyroidism presenting with spells of paroxysmal choreoathetosis is described. The possible mechanisms by which hypoparathyroidism induces choreoathetosis and other extrapyramidal motor dysfunctions are discussed. The need for screening patients with extrapyramidal disease for hypoparathyroidism is stressed.

Idiopathic hypoparathyroidism is a rare disorder often presenting with neuropsychiatric symptoms including mental retardation, emotional instability, anxiety, depression, confusional states, delusions, hallucinations, psychosis, and dementia (McKinney, 1962; Editorial, 1967; Fonesca and Calverley, 1967; Eraut, 1974). The common neurological manifestations are epileptic seizures, paraesthesias, and muscle cramps (Rose and Vas, 1966; Fonesca and Calverley, 1967; Hossain, 1970). Other neurological signs are benign intracranial hypertension, ataxia, cerebellar signs, dysarthria, dysphagia, and rarely extrapyramidal signs (Simpson, 1952; McKinney, 1962; Rose and Vas, 1966; Muentner and Whisnant, 1968; Hossain, 1970). We had the opportunity to study a patient whose only symptom of idiopathic hypoparathyroidism was paroxysmal choreoathetosis.

Our patient, an 18 year old girl, had a one year history of repeated spells of involuntary movements of her limbs and head lasting from a few minutes to several hours. When first seen at the neurological clinic she had a strange appearance, her behaviour was bizarre, and she was thought to be slightly mentally retarded. Her nails were thick and cracked, her hair thin and scanty, but no other abnormalities were found on general physical examination. Neurological examination was unremarkable, except for loss of the deep

${ }^{1}$ Address for reprint requests: Dr D. Soffer, Department of Neurology, Hadassah University Hospital, POB 499, Jerusalem, Israel.

Accepted 27 December 1976 tendon reflexes in the lower extremities. The electroencephalogram (EEG) was normal. Because of the bizarre behaviour, emotional instability, negative neurological examination, and normal EEG, the patient was thought to be suffering from a mental illness and was referred to a mental institution. While there, recurrent spells of involuntary movements were observed and she was admitted to the neurological department for re-evaluation.

On admission the patient was drowsy, unable to stand up without support, and her gait was unsteady. Spells of choreoathetotic movements, more prominent on the right side, were seen, associated with facial grimacing. Routine laboratory findings and liver function tests were normal. The Kahn and Wassermann reactions were negative. Lumbar puncture was normal except for elevated gammaglobulin fractions $(18.6 \%)$. Repeated EEG examinations revealed bursts of high amplitude slow and sharp waves activity on photic stimulation. Skull radiograph and isotope brain scan were normal. Ulnar nerve conduction velocity was normal. Repeated serum calcium was $4.0 \mathrm{mg} \mathrm{dl}$, serum phosphorus 7.0-7.7 mg dl, and serum magnesium $1 \mathrm{mg}$ dl. Other laboratory signs of hypocalcaemia were also found: In the ECG there were flat $T$ waves and prolonged QT interval, and the teeth were hypoplastic with enamel hypoplasia, resorption of alveolar bone, decalcification of jaws, and shortened teeth roots. Extensive laboratory investigations showed no evidence of renal disease, malabsorption, or other endocrine abnormalities. Skeletal radiological survey was normal, and 
tomography of the basal ganglia did not disclose calcifications. Injection of 200 units of parathormone intravenously increased urinary phosphate excretion by 2.4 times, confirming the diagnosis of idiopathic hypoparathyroidism (Ellsworth and Howard, 1934).

Treatment was begun with vitamin $\mathrm{D}$ and calcium. Gradually the patient's condition improved. She became alert, the involuntary movements stopped, the hair and nail changes disappeared, and the tendon reflexes reappeared. At present, after two years of treatment, she is free of symptoms and has married and given birth to a normal child.

\section{Discussion}

Extrapyramidal symptoms occur rarely in idiopathic hypoparathyroidism, often in association with basal ganglia calcifications. Muentner and Whisnant (1968) described 11 cases of extrapyramidal manifestations in idiopathic hypoparathyroidism and collected another 19 cases from the literature. Among the extrapyramidal syndromes which include parkinsonism, dystonia, torticollis, hemiballism, and oculogyric spasms (Simpson, 1952; McKinney, 1962; Fonesca and Calverley, 1967; Rose and Vas, 1966; Muentner and Whisnant, 1968; Hossain, 1970), chorea and athetosis are very rare. Since Simpson's (1952) description of the first case of chorea in idiopathic hypoparathyroidism, no more than 10 cases, including those with kinesiogenic choreiform movements, have been described in the literature (Tabaee-Zadeh et al., 1972; Christianson and Hansen, 1972). To our knowledge only one case of athetosis (Frankenberg and Rogers, 1959) aside from those of kinesiogenic choreoathetosis (Tabaee-Zadeh et al., 1972) has been described previously. The cause of the predilective and almost selective involvement of the basal ganglia in cases of hypoparathyroidism is unknown. Muentner and Whisnant (1968) suggested that the same noxious process which attacks the parathyroid glands attacks the basal ganglia as well. This mechanism, however, cannot explain the occurrence of extrapyramidal syndromes in post-operative hypoparathyroidism (McKinney, 1962; Fonesca and Calverley, 1967; Muentner and Whisnant, 1968; Rubenstein and Brust, 1974) and in hypocalcaemia secondary to other causes (Hosking et al., 1975). In most of the cases, correction of the basic metabolic disturbance reverse the neurological symptoms, even in those cases where basal ganglia calcification has already occurred. The fact that the neurological manifestations are reversible, while the calcifications are irreversible, suggests that calcification by itself is not the cause of the neurological impairment. Furthermore, there are cases of basal ganglia calcification without neurological manifestations (Bennett et al., 1959; Muentner and Whisnant, 1968; Smith et al., 1973), and cases of idiopathic hypoparathyroidism with extrapyramidal signs without basal ganglia calcifications. Therefore it is believed that calcifications are merely a reflection of long standing hypocalcaemia (McKinney, 1962; Smith et al., 1973), and the cause of the involuntary movements is a localised dysfunction of the basal ganglia induced by the hypocalcaemia. Others have suggested a vascular mechanism. Calcium tends to deposit within the walls of small vessels in the basal ganglia causing hyalinisation and degeneration of the vessels walls, resulting in hypoxic damage (Sugar, 1953; McKinney, 1962). Again, such a mechanism fails to explain the reversibility of the neurological symptomatology in the face of the irreversibility of the calcifications.

The most outstanding feature of our case was its presentation with spells of choreoathetosis. However, other neuropsychiatric features common in idiopathic hypoparathyroidism were also present including emotional instability, bizarre behaviour, arreflexia, and abnormal EEG. Depression of tendon reflexes may occur (Eaton and Haines, 1939; Rose and Vas, 1966) and is reversible with correction of hypocalcaemia, as seen also in this case. EEG abnormalities in addition to frank convulsions are common in idiopathic hypoparathyroidism and usually there is no correlation between the EEG changes and the serum level of calcium (Rose and Vas, 1966; Basser et al., 1969; Hossain, 1970).

Most of the neuropsychiatric manifestations of hypoparathyroidism could be explained by the neuronal hyperexcitability induced by hypocalcaemia. Calcium is a key component of the cell membrane, controlling its permeability and electrical properties. Hypocalcaemia increases membrane permeability, and as a result increases neuromuscular excitability (Rusmussen, 1974). Why the increased neuromuscular excitability manifests itself with different symptoms in different individuals is unknown.

This case stresses the need for screening for hypocalcaemia and hypoparathyroidism in every patient with choreoathetosis and other extrapyramidal signs of obscure origin. It is important to make the diagnosis of hypoparathyroidism, since specific treatment can cure the patient. 


\section{References}

Basser, L. S., Noale, F. C., Ireland, A. V., and Posen, S. (1969). Epilepsy and electroencephalographic abnormalities in chronic surgical hypoparathyroidism. Annals of Internal Medicine, 71, 505515.

Bennett, J. C., Maffly, R. H., and Steinbach, H. L. (1959). The significance of bilateral basal ganglia calcification. Radiology, 72, 368-377.

Christianson, N. J. B., and Hansen, P. F. (1972). Choreiform movements in hypoparathyroidism. New England Journal of Medicine, 287, 569-570.

Eaton, L. M., and Haines, S. F. (1939). Parathyroid insufficiency with symmetrical cerebral calcification. Journal of the American Medical Association, 113, 749-753.

Editorial (1967). Seizures and hypoparathyroidism. Journal of the American Medical Association, 201, 693.

Ellsworth, R., and Howard, J. E. (1934). Studies on the physiology of the parathyroid glands. Bulletin of the Johns Hopkins Hospital, 55, 296-308.

Eraut, D. (1974). Idicpathic hypoparathyroidism presenting as dementia. British Medical Journal, 1, 429-430.

Fonesca, O. A., and Calverley, J. R. (1967). Neurological manifestations of hypoparathyroidism. Archives of Internal Medicine, 120, 202-206.

Frankenberg, W. K., and Rogers, F. B. (1959). Hypoparathyroidism; review and case report. American Practitioner and Digest of Treatment, 10, 2130 2134.

Hosking, D. J., Williams, A., Godwin-Austen, R. B., and Allison, S. P. (1975). Osteomalacia presenting as chorea. British Medical Journal, 3, 136-138.
Hossain, M. (1970). Neurological and psychiatric manifestations in idiopathic hypoparathyroidism: response to treatment. Journal of Neurology, Neurosurgery, and Psychiatry, 33, 153-156.

McKinney, A. S. (1962). Idiopathic hypoparathyroidism presenting as chorea. Neurology (Minneapolis), 12, 485-491.

Muentner, M. D., and Whisnant, J. P. (1968). Basal ganglia calcification, hypoparathyroidism and extrapyramidal motor manifestations. Neurology (Minneapolis), 18, 1075-1083.

Rose, G. A., and Vas, C. J. (1966). Neurological ccmplications and electroencephalographic changes in hypoparathyroidism. Acta Neurologica Scandinavica, 42, 537-550.

Rubenstein, A., and Brust, J. C. M. (1974). Parkinsonian syndrome as complication of postthyroidectomy hypoparathyroidism. New York State Journal of Medicine, 75, 2029-2030.

Rusmussen, H. (1974). Parathyroid hormone, calcitonin and the calciferols. In Textbook of Endocrinology, 5th edition. Edited by R. H. Williams. W. B. Saunders: Philadelphia.

Simpson, J. A. (1952). The neurolcgical manifestations of idiopathic hypoparathyroidism. Brain, 75, 76-90.

Smith, K. D., Geraci, A., and Luparello, F. J. (1973). Basal ganglia calcification in postoperative hypoparathyroidism. New York State Journal of Medicine, 73, 1807-1809.

Sugar, O. (1953). Central neurological complications of hypoparathyroidism. Archives of Neurology and Psychiatry (Chicago), 70, 86-107.

Tabaee-Zadeh, M. J., Frame, B., and Kapphahn, K. (1972). Kinesiogenic choreoathetosis and idicpathic hypoparathyroidism. New England Journal of Medicine, 286, 762-763. 